\title{
Langzeitstudien zur medikamentösen Behandlung von Epilepsien im Kindesalter - eine komplexe Herausforderung
}

Follow-up Studies about Drug-Therapy of Epilepsies in Childhood - a Complex Challenge

H.-M. Straßburg

Bibliografie

DOI http://dx.doi.org/

$10.1055 / \mathrm{s}-0033-1349845$

Klin Padiatr 2013; 225: 245-246

(c) Georg Thieme Verlag KG

Stuttgart · New York

ISSN 0300-8630

Korrespondenzadresse

Prof. Dr. Hans Michael

Straßburg

Ehem. Universitäts-Kinderklinik

Würzburg

Emil-von-Behring-Weg 8

97218 Gerbrunn

strassburg@mail.uni-wuerzburg.de
Epilepsien haben aufgrund großer Fortschritte in Diagnostik und Therapie viel von ihrem früheren Schrecken verloren, dennoch ist für viele Eltern der erste zerebrale Anfall ihres Kindes ein besonders belastendes Ereignis. Auch sind die sich daraus ergebenden medizinischen Untersuchungen und Therapieüberlegungen für diejenigen, die mit der Materie nicht gut vertraut sind, oft nur schwer nachzuvollziehen.

Obwohl alte Befürchtungen, dass z.B. bei einem Anfall viele Tausende von Nervenzellen zerstört würden und ernste Lebensgefahr bestünde, seit Langem widerlegt sind, besteht unverändert ein deutlich erhöhtes Gesundheitsrisiko für Epilepsiepatienten bei bestimmten Tätigkeiten wie z.B. Schwimmen in offenen Gewässern, Klettern oder Führen von Fahrzeugen, im Kindes- und Jugendalter auch Fahrradfahren [5]. Neue Unsicherheiten entstehen, wenn ein Anfallsleiden auf dem Boden einer anderen Erkrankung entstanden ist. Die Zahl der Patienten mit anderen Grundkrankheiten, $z$. B. aus der Onkologie oder nach intensivmedizinischer Behandlung, ist gestiegen [6]. Hierbei dürfen allerdings die deutlich verbesserten Überlebensraten nicht ausgeblendet werden.

Insgesamt wird aufgrund der großen Fortschritte in der Diagnostik und der Therapie heute wesentlich seltener durch die verantwortlichen Neuropädiater die Indikation zur medikamentösen Dauerbehandlung gestellt als noch vor 20 Jahren. Die sachgerechte Aufklärung der Eltern und des Patienten sowie Änderungen der Lebensführung sind von besonderer Bedeutung, z.B. durch Schulungsprogramme, wie FAMOSES (www.famoses.de).

In ihrem Beitrag „Ambulante Betreuung von mit Antikonvulsiva behandelten Kindern - Fallstricke nach der Krankenhausentlassung“ haben die Autoren in einer sorgfältig geplanten Follow-upStudie den mittelfristigen Verlauf nach der Entlassung aus stationärer Behandlung analysiert [1]. Dabei stellte sich heraus, dass bereits innerhalb des Krankenhauses und im abschließenden Arztbrief, vor allem aber nach der Entlassung durch weiterbetreuende Ärzte, durch Apotheker und Eltern in erheblichem Umfang Veränderungen an der primär verordneten antikonvulsiven Medikation vorgenommen wurden. Außerdem bestanden bei über der Hälfte der Eltern erhebliche Wissensdefizite beim Umgang mit der Krankheit.

Dem Einwand, dass diese Ergebnisse Ausdruck einer schlechten Organisation, einer unzureichenden Infrastruktur oder eines mangelnden Kontaktes zwischen den neuropädiatrischen Spezialisten und den nachbetreuenden Einrich- tungen sei, muss allein schon aufgrund der Herkunft des Artikels, der beachtlichen Zahl an Probanden und ähnlicher Ergebnisse im internationalen Schrifttum widersprochen werden. Zudem wird über dieses Phänomen auch bei den akut lebensbedrohlichen Krebserkrankungen im Kindesalter berichtet [4].

Andererseits darf die beschriebene Situation sicher nicht fortbestehen, sodass auf verschiedenen Ebenen Veränderungen herbeizuführen sind, z.B. in Form von persönlich adaptierten Aufklärungsunterlagen und schriftlichen Dokumentationen, die kurzfristig überprüft werden. Kritiker der Studie sollten sich fragen, ob sie wirklich sicher sind, dass vergleichbare Verhältnisse bei ihnen nicht vorkommen.

Insgesamt zeigt die Studie, dass trotz vieler Fortschritte die Behandlung einer Epilepsie immer noch zu sehr von subjektiven Kriterien, Vorurteilen und von einem Kurieren am Symptom geprägt ist. Weiterhin gilt es, eine Reihe von offenen Fragen zu beantworten:

- Unverändert ist zu wenig über galenische Unterschiede bei den verschiedenen Medikationen, insbesondere auch im Vergleich von Markenartikeln und Fertigarzneimitteln bekannt.

- Letztlich wissen wir immer noch zu wenig über die spezifischen Mechanismen der antikonvulsiven Wirkung von Medikamenten. Viele Nebenwirkungen, z.B auch von häufig verordneten Medikamenten wie Valproat, Oxcarbazepin und Levetirazetam, sind immer noch nicht ausreichend erforscht $[2-5,7]$.

- Die Problematik, aktuelle und wissenschaftlichen Anforderungen standhaltende Daten zur Behandlung mit Antikonvulsiva zu erhalten, wurde u.a. auch bei der zwischen 2007-2009 durchgeführten HEAD-Studie deutlich, in der im Doppelblindversuch die Behandlung von BECTS (benign epilepsy with centro-temporal sharp waves) im Vergleich von Sultiam und Levetirazetam untersucht wurde (http:||clini caltrials.gov/show/NCT00471744). Trotz intensiver Bemühungen ist die anvisierte Probandenzahl nicht erreicht worden, zusätzlich gab es erhebliche Probleme mit dem zur Verfügung stellen der Studienmedikamente. Eine abschließende Publikation der Daten ist bis zum heutigen Tag nicht erfolgt [2].

- Leider besteht gerade auch in Deutschland eine große Zurückhaltung sowohl der Ärzte als auch der Eltern, bei Kindern mit einer Epilepsie kontrollierte Studien zur Effektivität und Effizienz einer Behandlung durchzuführen. So gibt es eine AWMF-Leitlinie zur Diagnostik, aber - von Ausnahmen abgesehen - 
keine offiziellen Leitlinien zu medikamentösen Therapie im Kindesalter [8].

- Vor allem fehlen aber multizentrische Daten, einschl. neuropsychologischer Befunde zur Langzeitprognose für die mittlerweile gut definierten Epilepsieformen [5].

Es ist zu wünschen, dass die in dem Beitrag vorgestellten Kommunikations- und Informationsprobleme allgemein weiter reduziert werden können. Darüber hinaus sind für die zunehmend kleiner werdende Gruppe von Kindern mit gravierender, therapierefraktärer Epilepsie möglichst effektive und nebenwirkungsarme Medikamente zu untersuchen, um auch für diese Kinder die Prognose und Lebensqualität zu verbessern.

\section{Literatur}

1 Bertsche A, Dahse AJ, Neininger $M$ et al. Ambulatory care of children treated with anticonvuldants - pitfalls after discharge from hospital. Klin Padiatr 2013; 225: 277-282

2 Borggräfe I, Bonfert M, Bast T et al. A double-blinded, randomized, head-to-head trial of levetiracetam versus sulthiam in benign epilepsy with centrotemporal spikes (Abstract). Neuropediatrics 2013; 44: 79

3 Borusiak $P$, Langer $T$, Heruth $M$ et al. Antiepileptic drugs and bone metabolism in children: data from 128 patients. J Child Neurol 2013; 28: $176-183$

4 Gottschling S, Längler A, Tautz C et al. Komplementäre und alternative Behandlungsmethoden in der Kinderonkologie. Klin Padiatr 2006; 218: $157-164$

5 Neubauer BA, Hahn A. Dooses Epilepsien im Kindes- und Jugendalter. 12. Auflage, Springer, Berlin: 2012

6 Tibussek D, Distelmaier S, Schönberger S et al. Antiepileptic treatment in paediatric oncology - an interdisciplinary challenge. Klin Padiatr 2006; 218: 340-349

7 Topf HG, Lischetzki G, Trollmann $R$ et al. The effect of valproat therapy on thrombin generation determines by calibrated automated thrombography. Klin Padiatr 2011; 223: 165-168

8 www.awmf.org/leitlinien/detail/II/022-007.htlm Diagnostische Prinzipien bei Epilepsien im Kindesalter 